\title{
Iron Deficiency Anemia in Children and Adolescents with Type I Diabetes, Is it a Real Problem?
}

\author{
ASMAA A. SOLIMAN, M.D.; SAFINAZ A. ELHABASHY, M.D.; RASHA A. THABET, M.D. and \\ NOURAN Y. SALAH EL DIN, M.B.B.Ch.
}

The Department of Pediatrics, Faculty of Medicine, Ain Shams University

\begin{abstract}
Background: Iron deficiency anemia (IDA) in children with type I diabetes (T1D) represents a significant burden.

Aim of Study: To asses iron status in children and adolescent with T1D of and to correlate it with glycemic control and diabetic vascular complications.

Patients and Methods: Two hundred children with T1D recruited from Pediatrics and Adolescent Diabetes Unit (PADU), Ain Shams University in the period from December 2019 to July 2020 . They were 123 males $(61.5 \%)$ and 77 females $(38.5 \%)$ aged $10.97 \pm 3.93$ years (Range: $2-18$ years). History taking, fundus examination and general examination were done stressing on anthropometric measurements. Laboratory evaluation including complete blood count, glycosylated haemoglobin (HbA1c), urinary albumin/creatinine ratio (ACR), lipid profile and patients with microcytic hypochromic anaemia underwent Serum iron, total iron-binding capacity (TIBC), serum ferritin, Hepcidin, Anti-tissue transglutaminase (IgA), Occult blood in stool and H-pylori antigen in stool.
\end{abstract}

Results: Seventy two of diabetic children were anemic (36\%) and fifty one had IDA (25.5). IDA was more prevalent in males. Children with T1D and IDA experienced more clinically significant hypoglycemic attacks, more DKA attacks, high fatigue severity scale and history of menorrhagia. Low body weight, low BMI, low mean corpuscular volume (MCV), high TIBC and low hepcidin level were present in diabetic children with IDA. They also had high HbA1c, neuropathy, high triglycerides and high level of low density lipoprotein (LDL cholesterol).

Conclusions: IDA is a significant morbidity among children with T1D and it should be screened. Serum hepcidin levels are significantly associated with iron status in children, and could be useful indicators of ID.

Key Words: Iron deficiency anemia - Children - Adolescents - Type I diabetes - Is it a Real Problem?.

Correspondence to: Dr. Asmaa A. Soliman, The Department of Pediatrics, Faculty of Medicine, Ain Shams University

\section{Introduction}

ANEMIA in type 1 diabetes (T 1 D) may have a complex, multifactorial background. Among the most common causes of anemia in the course of T1D in children is iron deficiency. Its prevalence is higher among T1D patients in comparison to people without diabetes [1].

Iron deficiency (ID) and iron deficiency anemia (IDA) can impair glucose homeostasis and may negatively affect glycemic control and predispose to more complications in diabetic patients [2].

Anemia is associated with an increased risk of diabetic complications including nephropathy, retinopathy and macro vascular disease. Anemia may also be significant in determining the outcome of heart failure and hypoxia-induced organ damage in diabetes. While several factors contribute to the increased prevalence of anemia in diabetes, the failure of the kidney to increase erythropoietin in response to falling hemoglobin appears to be the dominant factor [3].

On the other hand diabetes and its complications are associated with anemia and its correction improves diabetes control and may prevent or delay the occurrence of complications [2].

Iron replacement therapy decreases $\mathrm{HbA} 1 \mathrm{c}$ in both diabetic and non-diabetic individuals. This implies that the iron states must be considered during the interpretation of $\mathrm{HbA} 1 \mathrm{c}$ concentrations in diabetic or non-diabetic patients. Early diagnosis and treatment of ID in diabetic patients can improve their glycemic control and may prevent or delay complications [2]

Aim of the study:

To determine the frequency of iron deficiency anemia among type I diabetic children and to 
identify possible etiologies of iron deficiency anemia and to correlate different hematological parameters to glycemic control and diabetic vascular complications.

\section{Patients and Methods}

\section{Study design and sampling:}

A cross-sectional study was conducted on 200 children with T $1 \mathrm{D}$ aged from 2 to 18 years over 6 months from December 2019 to July 2020 at Pediatrics and Adolescent Diabetes Unit (PADU), Ain Shams University.

Sample size was calculated using pass program. Setting the type I error (a) at 0.05 of the confidence interval width at 0.2 (margin of error $10 \%$ ). Results from pervious study Thomas et al. [3] showed that $14 \%$ of DM cases had anemia. Calculation accordingly results in a minimal sample size of 47 approximated to 50 .

\section{Ethical considerations:}

Ethical approval and advice for study design and sample size was obtained from the Research Ethics Committee of Faculty of Medicine, Ain Shams University (FMASU REC). The FMASU REC is organized and operated according to guidelines of the International Council on Harmonization (ICH) Anesthesiology and the Islamic Organization for Medical Sciences (IOMS), the United States Office for Human Research Protection and the United States Code of Federal Regulations and operates under Federal wide (Assurance no. MS234/2020).

Parents or legal guardians were informed about the purpose and the anticipated benefits of the research and confidentiality of data was ensured. An informed consent in simple Arabic language was obtained from the legal guardians prior to enrollment in the study.

\section{Study population:}

Two hundred patients with T1D were recruited from Ain Shams University Pediatrics and Adolescent Diabetes Unit. Diagnosis of type I diabetes is based upon criteria of ISPAD 2018 [4].

Children with other types of diabetes e.g. Type 2 diabetes, Maturity onset diabetes of youth (MODY) and patients with any chronic disease affecting iron status such as autoimmune diseases, neoplasia, chronic kidney disease, and chronic liver disease were excluded from the study.
Study procedure:

All enrolled children were subjected to the following:

\section{1-Interview-based questionnaire:}

The main researcher interviewed the guardians to collect the following data:

\section{Personal data:}

Data on child's age, gender, order of birth, number of siblings, area of residence, father's and mother's educational level and occupation was collected to determine the socioeconomic status of study participants.

Socioeconomic status (SES) was assessed using the Modified scale for social level of families for usage in health research which was modified after the original scale of Fahmy and El-Sherbini where subjects were given a score from 54 and were further classified into 3 social classes; low, medium, or high, according to their score [5]

\section{Diabetes history:}

In terms of duration, and control over last two year prior study determined by frequency of hypoglycaemia and or diabetic ketoacidosis, history of fatigue using the Fatigue Severity Scale [6], history of parasitic infestations and history of menorrhagia in pubertal females.

\section{Dietetic history:}

Included type of feeding in early infancy; either exclusive breastfeeding, non-exclusive breast feeding (mixed feeding) or formula feeding, as well as a review of foods introduced during weaning, and a 24-hour recall of diet aimed at assessing the intake of required macro- and micro-nutrients to determine quality of diet.

Weaning was judged either to be faulty or correct depending on timing of its initiation and types of foods introduced, and according to WHO recommendations for infant feeding.

\section{Medical history:}

Parents were also asked if they noticed that their child experienced any symptoms of anemia such as development of pallor, easy fatigue, and lack of concentration, pica or palpitations and fatigue severity scale questionnaire was obtained from them [6].

\section{2- Examination:}

Anthropometric evaluation including weight in kilograms $(\mathrm{Kg})$, height in centimeters $(\mathrm{cm})$ and 
Body mass index (BMI) was calculated as follows weight $(\mathrm{kg}) /$ height $(\mathrm{m})^{2}$.

Weight, height and BMI Z scores was measured using $\mathrm{z}$ score reference for Egyptian children and adolescent [7].

Examination for tachycardia, radial pulse rate was performed a. Inspection for pallor at palms, nail beds, conjunctiva, oral mucosa and tongue was also done. Cardiac examination for hemic murmur and to exclude any underlying illness. Chest examination for dyspnea. Abdominal examination for hepato-splenomegaly. Slit-lamp biomicroscopy was done to evaluate retinopathy.

\section{3- Laboratory investigations:}

Complete Blood Count (CBC) was done for all subjects; Samples were collected by a trained nurse under complete aseptic condition. $2 \mathrm{ml}$ of blood were withdrawn into EDTA containing (purple top) tubes. CBC was performed using the Sysmex XT1800 i (Sysmex, Kobe, Japan) results will be interpreted according to special age and sex [8].

Glycated haemoglobin (HbA1c): A well-trained nurse had withdrawn the samples by pricking a finger and collecting finger capillary blood sample then the blood sample was added to the buffer and was shacked well to mix the blood with the buffer and through fluorescent immunochromotography analyzing system with fine care TM FIA meter plus device the result were collected.

Fasting lipid profile (triglycerides, total cholesterol, high density lipoproteins and low density lipoproteins which are done on Cobas c 111 (Roche Diagnostics, Mannheim, Germany) to detect dyslipidemia.

Patients with microcytic hypochromic anaemia underwent assessment of:

Serum iron and serum ferritin: $2 \mathrm{ml}$ of blood were with drawn into Gold-top serum separator tube (SST) and analysis was done using a 5010 spectrophotometer supplied by Roche diagnostics (GmbH, SandhoferStrasse 116, D-68305 Mannheim) [9].

Total iron-binding capacity (TIBC) measurement consists of three steps: The first step involves addition of supra physiological amounts of $\mathrm{FeCl} 3$ to saturate the free binding sites on transferrin; the second is the removal of unbound excess iron by adsorption onto solid magnesium carbonate, charcoal or an ion exchange resin; the third is the determination of iron that dissociated from trans- ferrin at acidic pH (done on Cobas c 111) (Roche Diagnostics, Mannheim, Germany).

Hepcidin: Venous blood samples of $2 \mathrm{ml}$ were collected. Separated serum was frozen to $70^{\circ}$ Celsius for storage and transport, and later thawed and analyzed in a single batch. Serum hepcidin was measured by a competitive enzyme-linked immunoassay (C-ELISA). Results from the CELISA were determined from standard curves developed from calibrators run simultaneously with study samples.

Anti-tissue transglutaminase (IgA): $1 \mathrm{ml}$ serum sample were collected in a red topped tube or gel barrier tube Serum anti-tissue transglutaminase IgA will be used to exclude celiac disease [10]. It will be measured with enzyme linked immunosorbent assay (ELISA) technique.

Occult blood in stool: It is used to look for active occult blood loss in anemia [11]. The stool samples were collected in a clean container and evaluated by detecting color changes on a test cardusing a microscope by immunochromatography technique.

$H$-pylori antigen in stool: Fresh fecal samples were collected in $60 \mathrm{ml}$ clean clearly labeled containers with wide mouths and screw-caps. Helicobacter Pylori Ag was measured by immunochromatography technique.

Assessment of diabetic chronic complication:

Diabetic neuropathy:

Diabetic neuropathy by performing simple rabid neuropathy test [12]

Diabetic nephropathy:

Albumin/creatinine ratio (ACR) in urine was examined to assess degree of diabetic nephropathy.

\section{Statistical analysis:}

Data were collected, revised, coded and entered to the Statistical Package for Social Science (IBM SPSS) version 23. The quantitative data were presented as mean, standard deviations and ranges when their distribution found parametric and median with inter-quartile range (IQR) when their distribution found non parametric. Also qualitative variables were presented as number and percentages.

The comparison between two groups regarding qualitative data were done by using Chi-square test and Fisher exact test instead of Chi-square test when the expected count in any cell found less than 5 . 
The comparison between two independent groups with quantitative data and non-parametric distribution were done by using Mann-Whitney test while the comparison between more than two groups was done by using Kruskall-Wallis test.

Spearman correlation coefficients were used to assess the correlation between two quantitative parameters in the same group.

Receiver operating characteristic curve (ROC) was used to assess the best cut off point with its sensitivity, specificity, positive predictive value (PPV), negative predictive value (NPV) and area under curve (AUC).

Logistic regression analysis was used to assess the relations with outcome with odds ratio and 95\% confidence interval (95\% CI).

Kaplan Mayer analysis was used to assess the relations with overall survival using Log Rank test.

The confidence interval was set to $95 \%$ and the margin of error accepted was set to 5\%. So, the pvalue was considered significant as the following:

- $p$-value $>0.05$ : Non significant (NS).

- $p$-value <0.05: Significant (S).

- $p$-value $<0.01$ : Highly significant (HS).

\section{Results}

The study was carried on 200 children with type 1 diabetes.

\section{Demographic data:}

The study included 200 children with T1D, IDA collectively was found in 51 children with percentage positivity $(25.5 \%)$.

Young male, clinically significant hypoglycemia, DKA and menorrhagia were found significantly higher in diabetic children with IDA than those without IDA.

Children with T1D and IDA had Low socioeconomic status, were formula fed, faulty and early weaned, less protein intake in a week, tachycardic and pale and had neuropathy.

Microcytosis, high HbA1c, Low serum iron, high TIBC, low hepcidin, high triglycerdies, high LDL cholesterol and presence of occult blood in stool were significantly present in diabetic children with IDA compared to those without IDA.

Positive correlation was found between IDA and male sex, lower age, hypoglycemic attacks, DKA attacks, positive menorrhagia history, fatigue severity scale, neuropathy, tachycardia, presence of pallor, low MCV, lower body weight, BMI lower than 19.93, TIBC more than 315, high HbA1c, low hepcidin, early faulty weaning, formula feeding, less times of protein intake, high level of LDL cholesterol and high triglycerides.

Table (1): Demographic and clinical characteristics of children with T1D $(n=200)$.

\begin{tabular}{|c|c|}
\hline & $\begin{array}{c}\text { Total } \\
\text { No. }=200\end{array}$ \\
\hline \multicolumn{2}{|l|}{ Gender: } \\
\hline Females & $77(38.5 \%)$ \\
\hline Males & $123(61.5 \%)$ \\
\hline \multicolumn{2}{|l|}{ Age (years): } \\
\hline Mean \pm SD & $10.97 \pm 3.93$ \\
\hline Range & $2-18$ \\
\hline \multicolumn{2}{|c|}{ Diabetes duration (years): } \\
\hline Median (IQR) & $2(15)$ \\
\hline Range & $1-15$ \\
\hline \multicolumn{2}{|c|}{ Insulin dose (IU/kg/day): } \\
\hline Mean \pm SD & $1.06 \pm 0.28$ \\
\hline Range & $0.5-2.2$ \\
\hline \multicolumn{2}{|c|}{ Clinically significant hypoglycemia/year: } \\
\hline Median (IQR) & $0(0-1)$ \\
\hline Range & $0-4$ \\
\hline \multicolumn{2}{|c|}{ Diabetic ketoacidosis/year: } \\
\hline Median (IQR) & $1(0-2)$ \\
\hline Range & $0-5$ \\
\hline \multicolumn{2}{|c|}{ Socioeconomic class: } \\
\hline Low & $77(38.5 \%)$ \\
\hline Medium & $100(50.0 \%)$ \\
\hline High & $23(11.5 \%)$ \\
\hline \multicolumn{2}{|l|}{ Early life feeding: } \\
\hline Breast fed. & $106(53.0 \%)$ \\
\hline Formula fed. & $43(21.5 \%)$ \\
\hline Mixed feeding & $51(25.5 \%)$ \\
\hline \multicolumn{2}{|l|}{ Weaning: } \\
\hline Correct & $136(68.0 \%)$ \\
\hline Faulty & $64(32.0 \%)$ \\
\hline \multicolumn{2}{|l|}{ Time of weaning: } \\
\hline Correct & $139(69.5 \%)$ \\
\hline Early & $43(21.5 \%)$ \\
\hline Late & $18(9.0 \%)$ \\
\hline \multicolumn{2}{|l|}{ Protein intake/week: } \\
\hline Median (IQR) & $3(2-3)$ \\
\hline & $1-7$ \\
\hline \multicolumn{2}{|c|}{ Multi-vitamins or fortified food intake: } \\
\hline No & $192(96.0 \%)$ \\
\hline Yes & $8(4.0 \%)$ \\
\hline \multicolumn{2}{|c|}{ Fatigue severity scale: } \\
\hline Median (IQR) & $2.2(1.8-3)$ \\
\hline Range & $1-6$ \\
\hline \multicolumn{2}{|l|}{ Neuropathy: } \\
\hline Negative & $179(89.5 \%)$ \\
\hline Positive & $21(10.5 \%)$ \\
\hline
\end{tabular}


Table (1): Cont.

\begin{tabular}{|c|c|}
\hline & $\begin{array}{c}\text { Total } \\
\text { No. }=200\end{array}$ \\
\hline \multicolumn{2}{|l|}{ Nephropathy: } \\
\hline Negative & $169(84.5 \%)$ \\
\hline Positive & $31(15.5 \%)$ \\
\hline \multicolumn{2}{|l|}{ Retinopathy: } \\
\hline Negative & $187(93.5 \%)$ \\
\hline Positive & $13(6.5 \%)$ \\
\hline \multicolumn{2}{|c|}{ Parasitic infestation history: } \\
\hline Negative & $152(76.0 \%)$ \\
\hline Positive & $48(24.0 \%)$ \\
\hline \multicolumn{2}{|l|}{ Menorrhagia history: } \\
\hline No menstruation & $31(15.5 \%)$ \\
\hline No menorrhagia & $33(16.5 \%)$ \\
\hline Menorrhagia & $13(6.5 \%)$ \\
\hline Males & $123(61.5 \%)$ \\
\hline \multicolumn{2}{|c|}{ Heart rate ( beat/minute): } \\
\hline Mean \pm SD & $89.35 \pm 8.29$ \\
\hline Range & $70-112$ \\
\hline \multicolumn{2}{|l|}{ Pallor: } \\
\hline Negative & $131(65.5 \%)$ \\
\hline Positive & $69(34.5 \%)$ \\
\hline \multicolumn{2}{|l|}{ Weight $(\mathrm{kg})$ : } \\
\hline Mean \pm SD & $36.88 \pm 15.47$ \\
\hline Range & $11-85$ \\
\hline \multicolumn{2}{|l|}{ Weight z-score: } \\
\hline Median (IQR) & $0(-1-1)$ \\
\hline Range & $-3-3$ \\
\hline \multicolumn{2}{|l|}{ Height $(\mathrm{cm})$ : } \\
\hline Mean \pm SD & $133.37 \pm 19.08$ \\
\hline Range & $84-175$ \\
\hline \multicolumn{2}{|l|}{ Height z-score: } \\
\hline Median (IQR) & $-1(-2-0)$ \\
\hline Range & $-3-3$ \\
\hline \multicolumn{2}{|c|}{ Body mass index (BMI): } \\
\hline Mean \pm SD & $19.94 \pm 5.00$ \\
\hline Range & $11.89-40.4$ \\
\hline \multicolumn{2}{|l|}{ BMI z-score: } \\
\hline Median (IQR) & $1(0-1)$ \\
\hline Range & $-3-3$ \\
\hline \multicolumn{2}{|l|}{$B M I:$} \\
\hline Underweight & $86(43.0 \%)$ \\
\hline Normal & $88(44.0 \%)$ \\
\hline Overweight & $18(9.0 \%)$ \\
\hline Obese & $8(4.0 \%)$ \\
\hline
\end{tabular}

Table (2): Laboratory data of the studied children with T1D $(n=200)$.

\begin{tabular}{|c|c|}
\hline & $\begin{array}{c}\text { Total } \\
\text { No. }=200\end{array}$ \\
\hline $\begin{array}{l}\text { Hemoglobin gm/dl: } \\
\text { Mean } \pm \text { SD } \\
\text { Range }\end{array}$ & $\begin{array}{l}12.10 \pm 1.77 \\
8-16.2\end{array}$ \\
\hline $\begin{array}{l}\text { Anemia: } \\
\text { No } \\
\text { Yes }\end{array}$ & $\begin{array}{l}128(64.0 \%) \\
72(36.0 \%)\end{array}$ \\
\hline $\begin{array}{l}\text { Types of anemia: } \\
\text { No anemia and no microcytosis } \\
\text { Non microcytic anemia } \\
\text { Microcytosis without anemia } \\
\text { Iron deficiency anemia }\end{array}$ & $\begin{array}{l}108(54.0 \%) \\
21(10.5 \%) \\
20(10.0 \%) \\
51(25.5 \%)\end{array}$ \\
\hline $\begin{array}{l}\text { Mean corpuscular volume fl: } \\
\text { Mean } \pm \mathrm{SD} \\
\text { Range }\end{array}$ & $\begin{array}{l}75.96 \pm 8.33 \\
50-99.8\end{array}$ \\
\hline $\begin{array}{l}\text { Red cell distribution width } \% \text { : } \\
\text { Mean } \pm \text { SD } \\
\text { Range }\end{array}$ & $\begin{array}{l}14.14 \pm 1.78 \\
11-21\end{array}$ \\
\hline $\begin{array}{l}\text { Glycated hemoglobin }(\text { Hbalc }) \% \text { : } \\
\text { Mean } \pm \text { SD } \\
\text { Range }\end{array}$ & $\begin{array}{l}9.18 \pm 1.17 \\
7.4-13\end{array}$ \\
\hline $\begin{array}{l}\text { Serum ferritin } n g / d l \text { : } \\
\text { Median (IQR) } \\
\text { Range }\end{array}$ & $\begin{array}{l}45(25-78) \\
10-350\end{array}$ \\
\hline $\begin{array}{l}\text { Serum ferritin level: } \\
\quad \text { Low normal }<60 \\
\quad \text { Normal }\end{array}$ & $\begin{array}{l}47(66.2 \%) \\
24(33.8 \%)\end{array}$ \\
\hline $\begin{array}{l}\text { Serum iron } m g / d l \text { : } \\
\quad \text { Mean } \pm \mathrm{SD} \\
\text { Range }\end{array}$ & $\begin{array}{l}79.00 \pm 24.36 \\
30-120\end{array}$ \\
\hline $\begin{array}{l}\text { Total iron binding capacity }(T I B C) m g / d l: \\
\text { Mean } \pm \mathrm{SD} \\
\text { Range }\end{array}$ & $\begin{array}{l}316.18 \pm 66.16 \\
165-455\end{array}$ \\
\hline $\begin{array}{l}\text { Hepcidin pg/dl: } \\
\text { Median (IQR) } \\
\text { Range }\end{array}$ & $\begin{array}{l}1300(850-2900) \\
550-4800\end{array}$ \\
\hline $\begin{array}{l}\text { Anti-tissue transglutaminase IgA eu/ml: } \\
\text { Median (IQR) } \\
\text { Range }\end{array}$ & $\begin{array}{l}7(5-9) \\
1-14\end{array}$ \\
\hline $\begin{array}{l}\text { Occult blood: } \\
\text { Negative } \\
\text { Weak positive } \\
\text { Positive }\end{array}$ & $\begin{array}{l}68(97.1 \%) \\
2(2.9 \%) \\
0(0.0 \%)\end{array}$ \\
\hline $\begin{array}{l}H \text {-pylori antigen in stool: } \\
\text { Negative } \\
\text { Weak positive } \\
\text { Positive }\end{array}$ & $\begin{array}{l}56(80.0 \%) \\
10(14.3 \%) \\
4(5.7 \%)\end{array}$ \\
\hline $\begin{array}{l}\text { Cholesterol: } \\
\quad \text { Mean } \pm \mathrm{SD} \\
\quad \text { Range }\end{array}$ & $\begin{array}{l}163.41 \pm 28.84 \\
93-238\end{array}$ \\
\hline $\begin{array}{l}\text { HDL cholesterol: } \\
\text { Mean } \pm \mathrm{SD} \\
\text { Range }\end{array}$ & $\begin{array}{l}65.00 \pm 18.81 \\
25-141\end{array}$ \\
\hline $\begin{array}{l}\text { LDL cholesterol: } \\
\text { Mean } \pm \mathrm{SD} \\
\text { Range }\end{array}$ & $\begin{array}{l}92.80 \pm 23.52 \\
45-164\end{array}$ \\
\hline $\begin{array}{l}\text { Triglyceride: } \\
\quad \text { Mean } \pm \text { SD } \\
\quad \text { Range }\end{array}$ & $\begin{array}{l}74.50 \pm 27.94 \\
34-213\end{array}$ \\
\hline
\end{tabular}


Table (3): Comparison between diabetic children with IDA and without IDA in clinical and demographic data.

\begin{tabular}{|c|c|c|c|c|}
\hline & $\begin{array}{c}\text { No iron deficiency } \\
\text { anemia (IDA) } \\
\text { No. }=149\end{array}$ & $\begin{array}{c}\text { Iron deficiency } \\
\text { anemia } \\
\text { No. }=51\end{array}$ & $\begin{array}{c}\text { Test } \\
\text { value }\end{array}$ & $\begin{array}{c}p- \\
\text { value }\end{array}$ \\
\hline \multicolumn{5}{|l|}{ Gender: } \\
\hline Females & $69(46.3 \%)$ & $8(15.7 \%)$ & $15.048 *$ & $<0.001$ \\
\hline Males & $80(53.7 \%)$ & $43(84.3 \%)$ & & \\
\hline \multicolumn{5}{|l|}{ Age (years): } \\
\hline Mean \pm SD & $11.33 \pm 3.91$ & $9.91 \pm 3.84$ & $2.246 \bullet$ & 0.026 \\
\hline Range & $2-18$ & $4-18$ & & \\
\hline \multicolumn{5}{|c|}{ Diabetes duration (years): } \\
\hline Median (IQR) & $2(1-4)$ & $3(1.5-5)$ & $-1.571 \neq$ & 0.116 \\
\hline Range & $1-15$ & $1-11$ & & \\
\hline \multicolumn{5}{|c|}{ Insulin dose (unit/kg/day): } \\
\hline Mean \pm SD & $1.05 \pm 0.30$ & $1.06 \pm 0.21$ & $-0.108 \bullet$ & 0.914 \\
\hline Range & $0.5-2.2$ & $0.5-1.4$ & & \\
\hline \multicolumn{5}{|c|}{ Clinically significant hypoglycemia/year: } \\
\hline Median (IQR) & $0(0-0)$ & $2(0-2)$ & $-6.883 \neq$ & $<0.001$ \\
\hline Range & $0-3$ & $0-4$ & & \\
\hline \multicolumn{5}{|c|}{ Diabetic ketoacidosis/year: } \\
\hline Median (IQR) & $1(0-1)$ & $2(1-3)$ & $-5.883 \neq$ & $<0.001$ \\
\hline Range & $0-4$ & $0-5$ & & \\
\hline \multicolumn{5}{|c|}{ Parasitic infestation history: } \\
\hline Negative & $110(73.8 \%)$ & $42(82.4 \%)$ & $1.515^{*}$ & 0.218 \\
\hline Positive & $39(26.2 \%)$ & $9(17.6 \%)$ & & \\
\hline \multicolumn{5}{|l|}{ Menorrhagia history: } \\
\hline No menstruation & $29(19.5 \%)$ & $2(3.9 \%)$ & 21.634 & $<0.001$ \\
\hline No menorrhagia & $32(21.5 \%)$ & $1(2.0 \%)$ & & \\
\hline Menorrhagia & $8(5.4 \%)$ & $5(9.8 \%)$ & & \\
\hline Males & $80(53.7 \%)$ & $43(84.3 \%)$ & & \\
\hline \multicolumn{5}{|l|}{ Socioeconomic class: } \\
\hline Low & $31(20.8 \%)$ & $46(90.2 \%)$ & $77.513^{*}$ & $<0.001$ \\
\hline Medium & $95(63.8 \%)$ & $5(9.8 \%)$ & & \\
\hline High & $23(15.4 \%)$ & $0(0.0 \%)$ & & \\
\hline \multicolumn{5}{|l|}{ Early life feeding: } \\
\hline Breast fed. & $100(67.1 \%)$ & $6(11.8 \%)$ & $71.202 *$ & $<0.001$ \\
\hline Formula fed. & $12(8.1 \%)$ & $31(60.8 \%)$ & & \\
\hline Mixed feeding & $37(24.8 \%)$ & $14(27.5 \%)$ & & \\
\hline \multicolumn{5}{|l|}{ Weaning: } \\
\hline Correct & $126(84.6 \%)$ & $10(19.6 \%)$ & $73.672 *$ & $<0.001$ \\
\hline Faulty & $23(15.4 \%)$ & $41(80.4 \%)$ & & \\
\hline \multicolumn{5}{|l|}{ Time of weaning: } \\
\hline Correct & $126(84.6 \%)$ & $13(25.5 \%)$ & $62.569^{*}$ & $<0.001$ \\
\hline Early & $16(10.7 \%)$ & $27(52.9 \%)$ & & \\
\hline Late & $7(4.7 \%)$ & $11(21.6 \%)$ & & \\
\hline \multicolumn{5}{|c|}{ Protein intake in a week: } \\
\hline \multirow[t]{2}{*}{ Median (IQR) } & $3(3-4)$ & $2(1-2)$ & $-8.396 \neq$ & $<0.001$ \\
\hline & $1-7$ & $1-3$ & & \\
\hline \multicolumn{5}{|c|}{ Multi-vitamins or fortified food intake: } \\
\hline No & $141(94.6 \%)$ & $51(100.0 \%)$ & $2.852^{*}$ & 0.091 \\
\hline Yes & $8(5.4 \%)$ & $0(0.0 \%)$ & & \\
\hline \multicolumn{5}{|c|}{ Fatigue severity scale: } \\
\hline Median (IQR) & $2(1.8-2.5)$ & $3(2-4.5)$ & $-4.635 \neq$ & $<0.001$ \\
\hline Range & $1-5$ & $1-6$ & & \\
\hline
\end{tabular}


Table (3): Cont.

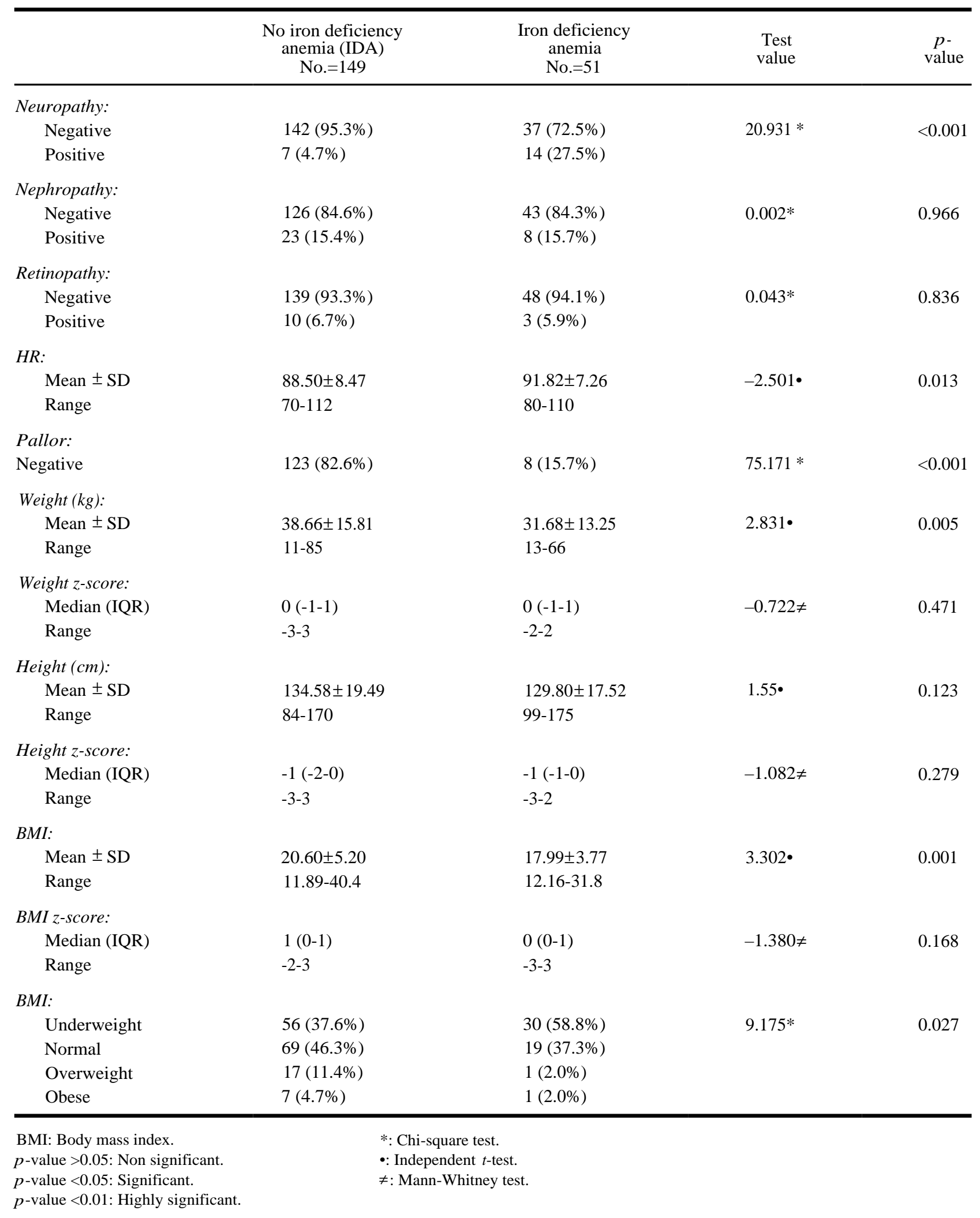


Table (4): Laboratory difference among diabetic children with and without IDA.

\begin{tabular}{|c|c|c|c|c|}
\hline & $\begin{array}{c}\text { No iron deficiency } \\
\text { anemia (IDA) } \\
\text { No. }=20\end{array}$ & $\begin{array}{l}\text { Iron deficiency } \\
\text { anemia } \\
\text { No.=51 }\end{array}$ & $\begin{array}{c}\text { Test } \\
\text { value }\end{array}$ & $\begin{array}{c}p- \\
\text { value }\end{array}$ \\
\hline \multicolumn{5}{|l|}{ Serum ferritin $\mathrm{ng} / \mathrm{ml}$ : } \\
\hline Median (IQR) & $50(32.5-60)$ & $45(20-80)$ & $-0.935 \neq$ & 0.350 \\
\hline Range & $10-250$ & $10-350$ & & \\
\hline \multicolumn{5}{|l|}{ Serum Iron ug/ml: } \\
\hline Mean \pm SD & $96.95 \pm 17.86$ & $71.96 \pm 23.02$ & $4.360 \bullet$ & $<0.001$ \\
\hline Range & $55-120$ & $30-115$ & & \\
\hline \multicolumn{5}{|l|}{ TIBC ug/ml: } \\
\hline Mean \pm SD & $280.90 \pm 35.15$ & $330.02 \pm 70.47$ & $-2.967 \bullet$ & 0.004 \\
\hline Range & $165-341$ & $213-455$ & & \\
\hline \multicolumn{5}{|l|}{ Hepcidin pg/ml: } \\
\hline Median (IQR) & $2900(2450-3180)$ & $1000(750-2100)$ & $-4.234 \neq$ & $<0.001$ \\
\hline Range & $750-4800$ & $550-4200$ & & \\
\hline \multicolumn{5}{|c|}{ Anti-tissue transglutaminase eu/ml: } \\
\hline Median (IQR) & $8(5-9)$ & $7(5-9)$ & $-0.597 \neq$ & 0.550 \\
\hline Range & $3-10$ & $1-14$ & & \\
\hline \multicolumn{5}{|l|}{ Occult blood: } \\
\hline Negative & $18(90.0 \%)$ & $51(100.0 \%)$ & $5.248 *$ & 0.022 \\
\hline Weak positive & $2(10.0 \%)$ & $0(0.0 \%)$ & & \\
\hline Positive & $0(0.0 \%)$ & $0(0.0 \%)$ & & \\
\hline \multicolumn{5}{|c|}{ H-pylori antigen in stool: } \\
\hline Negative & $15(75.0 \%)$ & $42(82.4 \%)$ & $0.808^{*}$ & 0.668 \\
\hline Weak positive & $4(20.0 \%)$ & $6(11.8 \%)$ & & \\
\hline Positive & $1(5.0 \%)$ & $3(5.9 \%)$ & & \\
\hline \multicolumn{5}{|l|}{$M C V f l:$} \\
\hline Mean \pm SD & $79.38 \pm 5.74$ & $65.96 \pm 6.44$ & $13.950 \bullet$ & $<0.001$ \\
\hline Range & $58.3-99.8$ & $50-80$ & & \\
\hline \multicolumn{5}{|l|}{$R D W \%:$} \\
\hline Mean \pm SD & $14.01 \pm 1.74$ & $14.52 \pm 1.85$ & $-1.763 \bullet$ & 0.079 \\
\hline Range & $11-21$ & $12-20.7$ & & \\
\hline \multicolumn{5}{|l|}{ HbAlc \%: } \\
\hline Mean \pm SD & $8.84 \pm 0.94$ & $10.16 \pm 1.23$ & $-7.965 \bullet$ & $<0.001$ \\
\hline Range & $7.4-12$ & $8-13$ & & \\
\hline \multicolumn{5}{|l|}{ Cholesterol: } \\
\hline Mean \pm SD & $163.36 \pm 28.99$ & $163.55 \pm 28.68$ & $-0.041 \bullet$ & 0.967 \\
\hline Range & $96-238$ & $93-225$ & & \\
\hline \multicolumn{5}{|l|}{ HDL cholesterol: } \\
\hline Mean \pm SD & $65.60 \pm 18.96$ & $63.25 \pm 18.46$ & $0.767 \bullet$ & 0.444 \\
\hline Range & $25-137$ & $37-141$ & & \\
\hline \multicolumn{5}{|l|}{ LDL cholesterol: } \\
\hline Mean \pm SD & $90.62 \pm 24.08$ & $99.18 \pm 20.75$ & $-2.266 \bullet$ & 0.025 \\
\hline Range & $45-164$ & $60-155$ & & \\
\hline \multicolumn{5}{|l|}{ Triglyceride: } \\
\hline Mean \pm SD & $72.12 \pm 26.47$ & $81.43 \pm 31.12$ & $-2.071 \bullet$ & 0.040 \\
\hline Range & $34-196$ & $35-213$ & & \\
\hline
\end{tabular}

TIBC: Total iron binding capacity. MCV: Mean corpuscular volume. RDW: Red cell distribution width. HbA1c: Glycated hemoglobin. HDL: High density lipoprotein. LDL: Low density lipoprotein. $p$-value $>0.05$ : Non significant. $p$-value $<0.05$ : Significant.

$p$-value <0.01: Highly significant.

*: Chi-square test.

$\bullet:$ Independent $t$-test.

$\neq$ : Mann-Whitney test. 
Table (5): Univar ate logistic regression analysis for factors associated with IDA.

\begin{tabular}{|c|c|c|c|c|c|c|c|}
\hline & \multirow{2}{*}{$\mathrm{B}$} & \multirow{2}{*}{ S.E. } & \multirow{2}{*}{ Wald } & \multirow{2}{*}{$\begin{array}{c}p- \\
\text { value }\end{array}$} & \multirow{2}{*}{$\begin{array}{l}\text { Odds ratio } \\
\quad(\mathrm{OR})\end{array}$} & \multicolumn{2}{|c|}{$95 \%$ C.I. for (OR) } \\
\hline & & & & & & Lower & Upper \\
\hline Sex (males) & 1.534 & 0.419 & 13.425 & $<0.001$ & 4.636 & 2.041 & 10.531 \\
\hline Age (years) $<10$ & 1.085 & 0.338 & 10.300 & 0.001 & 2.959 & 1.526 & 5.740 \\
\hline LDL cholesterol $>80$ & 1.231 & 0.420 & 8.591 & 0.003 & 3.426 & 1.504 & 7.805 \\
\hline Triglyceride $>82$ & 1.033 & 0.337 & 9.373 & 0.002 & 2.809 & 1.450 & 5.440 \\
\hline Hypoglycemic coma >1 & 2.623 & 0.400 & 43.054 & 0.000 & 13.776 & 6.293 & 30.155 \\
\hline Diabetic ketoacidosis times $>1$ & 2.164 & 0.369 & 34.342 & 0.000 & 8.707 & 4.222 & 17.957 \\
\hline Neuropathy & 0.697 & 0.349 & 3.986 & 0.046 & 2.008 & 1.013 & 3.982 \\
\hline $\mathrm{HR}>84$ & 1.421 & 0.467 & 9.253 & 0.002 & 4.141 & 1.658 & 10.343 \\
\hline Pallor & 3.236 & 0.441 & 53.738 & $<0.001$ & 25.428 & 10.705 & 60.401 \\
\hline $\mathrm{MCV}<75.4$ & 5.562 & 1.034 & 28.924 & $<0.001$ & 260.417 & 34.302 & 1977.065 \\
\hline Weight $(\mathrm{kg})<30$ & 1.171 & 0.339 & 11.934 & 0.001 & 3.225 & 1.660 & 6.268 \\
\hline $\mathrm{HbA} 1 \mathrm{c}>9.4$ & 2.251 & 0.380 & 35.102 & $<0.001$ & 9.493 & 4.509 & 19.988 \\
\hline BMI $<19.39$ & 1.050 & 0.349 & 9.081 & 0.003 & 2.859 & 1.444 & 5.661 \\
\hline S Iron $<75$ & -0.414 & 0.641 & 0.418 & 0.518 & 0.661 & 0.188 & 2.321 \\
\hline TIBC $>315$ & 2.473 & 0.797 & 9.627 & 0.002 & 11.864 & 2.487 & 56.595 \\
\hline Hepcidin $<1900$ & 2.807 & 0.704 & 15.908 & $<0.001$ & 16.564 & 4.169 & 65.808 \\
\hline Formula feeding & 0.804 & 0.196 & 16.747 & $<0.001$ & 2.234 & 1.520 & 3.283 \\
\hline Faulty weaning & 3.112 & 0.419 & 55.079 & $<0.001$ & 22.461 & 9.875 & 51.089 \\
\hline Early time of weaning & 1.770 & 0.288 & 37.775 & $<0.001$ & 5.868 & 3.337 & 10.317 \\
\hline Protein intake in a week $<2$ & 3.060 & 0.436 & 49.274 & $<0.001$ & 21.321 & 9.074 & 50.099 \\
\hline
\end{tabular}

CI: Confidence interval.

Table (6): Multivariate logistic regression analysis for factors associated with IDA.

\begin{tabular}{|c|c|c|c|c|c|c|c|}
\hline & \multirow{2}{*}{$\mathrm{B}$} & \multirow{2}{*}{ S.E. } & \multirow{2}{*}{ Wald } & \multirow{2}{*}{$\begin{array}{c}p- \\
\text { value }\end{array}$} & \multirow{2}{*}{$\begin{array}{l}\text { Odds ratio } \\
\quad(\mathrm{OR})\end{array}$} & \multicolumn{2}{|c|}{ 95\% C.I. for (OR) } \\
\hline & & & & & & Lower & Upper \\
\hline Hepcidin $<1900$ & 2.950 & 0.901 & 10.725 & 0.001 & 19.104 & 3.269 & 111.652 \\
\hline Neuropathy & -0.632 & 1.119 & 0.320 & 0.572 & 0.531 & 0.059 & 4.761 \\
\hline $\mathrm{HbA} 1 \mathrm{c}>9.4$ & 1.510 & 0.784 & 3.711 & 0.054 & 4.525 & 0.974 & 21.023 \\
\hline Diabetic ketoacidosis times $>1$ & 1.661 & 1.013 & 2.692 & 0.101 & 5.267 & 0.724 & 38.326 \\
\hline LDL cholesterol $>80$ & 2.050 & 1.110 & 3.410 & 0.065 & 7.764 & 0.882 & 68.373 \\
\hline Triglyceride $>82$ & 0.571 & 0.837 & 0.466 & 0.495 & 1.771 & 0.343 & 9.131 \\
\hline
\end{tabular}


Table (7): Correlation between factors related to IDA and TID.

\begin{tabular}{|c|c|c|c|c|c|c|c|c|}
\hline & \multicolumn{2}{|c|}{ S F } & \multicolumn{2}{|c|}{ S Iron } & \multicolumn{2}{|c|}{ TIBC } & \multicolumn{2}{|c|}{ Hepcidin } \\
\hline & $r$ & $p$-value & $r$ & $p$-value & $r$ & $p$-value & $r$ & $p$-value \\
\hline S F & & & 0.029 & 0.812 & $-.014-$ & 0.907 & 0.053 & 0.662 \\
\hline S Iron & 0.029 & 0.812 & & & $-0.797 * *$ & 0.000 & $.904 * *$ & 0.000 \\
\hline TIBC & -0.014 & 0.907 & $-0.797 * *$ & 0.000 & & & $-0.709 * *$ & 0.000 \\
\hline Hepcidin & 0.053 & 0.662 & $0.904 * *$ & 0.000 & $-0.709 * *$ & 0.000 & & \\
\hline Age (years) & -0.103 & 0.392 & 0.022 & 0.854 & -0.053 & 0.660 & 0.020 & 0.867 \\
\hline Cholesterol & 0.122 & 0.309 & 0.114 & 0.343 & -0.041 & 0.736 & 0.131 & 0.277 \\
\hline HDL cholesterol & -0.07 & 0.561 & -0.001 & 0.996 & 0.022 & 0.854 & -0.051 & 0.671 \\
\hline LDL cholesterol & -0.031 & 0.801 & 0.082 & 0.495 & 0.003 & 0.98 & 0.098 & 0.414 \\
\hline Triglyceride & 0.062 & 0.61 & 0.117 & 0.331 & -0.044 & 0.718 & 0.116 & 0.334 \\
\hline Diabetes duration (years) & 0.046 & 0.705 & $-0.307 * *$ & 0.009 & $0.235^{*}$ & 0.049 & -0.223 & 0.062 \\
\hline Insulin dose (KG/day) & 0.201 & 0.096 & -0.124 & 0.305 & 0.053 & 0.665 & -0.047 & 0.699 \\
\hline Hypoglycemic coma & $0.251 *$ & 0.035 & $-0.292 *$ & 0.014 & $0.246^{*}$ & 0.039 & $-0.285^{*}$ & 0.016 \\
\hline Diabetic ketoacidosis times & 0.152 & 0.205 & $-0.240 *$ & 0.044 & 0.183 & 0.126 & -0.211 & 0.077 \\
\hline Fatigue severity scale & -0.072 & 0.550 & $-0.417 * *$ & 0.000 & $0.299 *$ & 0.011 & $-0.430 * *$ & 0.000 \\
\hline $\mathrm{HR}$ & -0.020 & 0.867 & -0.091 & 0.450 & 0.063 & 0.600 & -0.066 & 0.583 \\
\hline Weight (kg) & -0.134 & 0.265 & -0.010 & 0.935 & -0.040 & 0.741 & -0.020 & 0.866 \\
\hline Weight z-score & $-0.400 * *$ & 0.001 & -0.170 & 0.158 & 0.135 & 0.261 & -0.158 & 0.188 \\
\hline Height $(\mathrm{cm})$ & -0.035 & 0.771 & 0.066 & 0.582 & -0.118 & 0.326 & 0.071 & 0.557 \\
\hline Height z-score & 0.048 & 0.691 & 0.042 & 0.729 & -0.065 & 0.588 & 0.015 & 0.903 \\
\hline BMI & -0.178 & 0.137 & -0.078 & 0.517 & 0.071 & 0.558 & -0.103 & 0.394 \\
\hline BMI z-score & -0.021 & 0.860 & -0.196 & 0.102 & 0.217 & 0.069 & -0.193 & 0.107 \\
\hline HGB & -0.107 & 0.377 & $0.599 * *$ & 0.000 & $-0.437 * *$ & 0.000 & $0.546 * *$ & 0.000 \\
\hline $\mathrm{MCV}$ & -0.231 & 0.053 & $0.473 * *$ & 0.000 & $-0.517 * *$ & 0.000 & $0.431 * *$ & 0.000 \\
\hline RDW & -0.096 & 0.427 & $-0.329 * *$ & 0.005 & $0.358 * *$ & 0.002 & $-0.237 *$ & 0.046 \\
\hline $\mathrm{HbA1c}$ & 0.183 & 0.126 & $-0.540 * *$ & 0.000 & $0.397 * *$ & 0.001 & $-0.481 * *$ & 0.000 \\
\hline Protein intake in aweek & -0.127 & 0.290 & $0.556^{* *}$ & 0.000 & $-0.413 * *$ & 0.000 & $0.514 * *$ & 0.000 \\
\hline Anti-tissue transglutaminase & 0.032 & 0.795 & 0.132 & 0.275 & -0.021 & 0.861 & 0.066 & 0.588 \\
\hline
\end{tabular}

\section{Discussion}

Type I diabetes (T1D) is one of the most common chronic diseases of childhood and is caused by immune-associated destruction of insulinproducing pancreatic beta-cells. Iron deficiency (ID) is common in children with T1D. Since ID in children is associated with adverse effects, such as cognitive and behavioral impairment, it is important to assess their iron status [13]

Until present, there have not been many studies assessing the prevalence of anemia among children with T1D. There is a range of publications regarding patients with type 2 diabetes and anemia, usually accompanying renal complications.

Analysis of the collected data in this study revealed that, the overall prevalence rate of anemia among 200 children with type I diabetes is $36 \%$ while Prevalence rate of iron deficiency anemia among 200 children with type I diabetes is $25.5 \%$ this result is similar to that of Wójciak et al. [14] who studied 100 children with type I diabetes, aged 6 to 17 years and found that the incidence of iron deficiency anemia is $26 \%$ in newly diagnosed children with type I diabetes.

While in an analysis of 200 children with T1D conducted in Egypt, anemia was diagnosed in 37\% of cases. Among patients with anemia $54.7 \%$ had iron deficiency [15].

Also in a cross sectional study including 109 people with type I diabetes $(54.1 \%$ male, mean age 56.2 years) at the diabetes clinic of the Goethe University Hospital. Decreased serum iron and ferritin levels were observed in $18(16.8 \%)$ and 28 (26.7\%) patients, respectively. Anemia was present in 20 patients $18.34 \%$ [16]

While a two-center prospective observational study in which the iron status of Dutch children with DM type I was determined during a regular check-up. Absolute iron deficiency and functional iron deficiency were found in 13/227 (5.7\%) and $100 / 214(47 \%)$ patients, respectively, while only $15 / 113(13 \%)$ patients also had anemia [17]. 


\section{Demographic data:}

In our study low socioeconomic class was highly significantly present in diabetic children with IDA than those without IDA this is similar to Abdel-Rasoul et al. [18], who find that children from low and middle socioeconomic standard had a two-fold increased risk of developing IDA [OR $1.59,95 \%$ confidence interval (CI) 1.1-2.4]. This is in agreement with the study carried out by Neuman et al. [19] in Brazil, in which IDA was more prevalent in children from low socioeconomic backgrounds. This can be as attributed to the fact that poverty is a contributing factor to IDA because families living at or below the poverty line may not be consuming enough iron-rich foods.

In our study from 200 patient there were 123 male $(61.5 \%)$ and 77 patients $(38.5 \%)$ were females. Their ages ranged from 2 years to 18 years with mean age $10.9 \pm 3.93$ years $(S D)$. Median duration of T $1 \mathrm{D}$ was 2 (1-5) years IQR.

On the other side, a study was done by Wójciak et al. [14] on 94 children with T1D (55 females) $58.5 \%$ and 39 males $41.4 \%$ at Diabetic Outpatient Clinic, Upper Silesian Centre for Child's Health in Katowice, Poland. Their mean age at study time was $12.5 \pm 4.1$ years (SD) (ranging from 3 to 19 years). Mean duration of T1D was $4.2 \pm 3.6$ years (SD).

In our study IDA was more common in males than in female this was statistically highly significant ( $p$-value 0.000$)$.

This is consistent with the results of Solomon et al. [20] who studied 87 patients diagnosed with IDA, where 53 (60.9\%) were male and 34 (39.1\%) were female. Of the 87 non-IDA diabetic patients, $51(58.6 \%)$ were female and $36(41.4 \%)$ were male.

While according to Andriastuti et al. [21] who studied two groups children age group and the adolescent and found that there were a total of 45 subjects in the children age group, which included 21 females (46.7\%) and 24 males (53.3\%). Twenty percent (nine subjects) had anemia. The overall prevalence of IDA, ID, and iron depletion was $11.1 \%, 15.6 \%$, and $4 \%$, respectively. The prevalence of anemia and iron deficiency was higher in the male population.

The adolescent group consisted of 162 subjects, and $51.2 \%$ were females and $48.8 \%$ were males. The prevalence of anemia, IDA, ID, and iron depletion was higher in the female group.

He defines school-aged children as children aged 6-9 years old; we describe adolescents as young people between the age of 10 and 18 years old [21]

On the other hand Abdel-Rasoul et al. [18] found that IDA occurred in both sexes, but more among females (57.5\%), although no significant difference found; this in agreement with Mohamed et al. [22] No sex differences were found in the prevalence of anemia, although a slight difference was noted $(12.8 \%)$ for girls and for boys $(11.4 \%)$. This may be due to unhealthy food consumption and blood loss during menstruation in older girls.

\section{Dietetic history:}

In this study we find that formula feeding, faulty and early weaning were found significantly higher in diabetics with iron deficiency anemia than in diabetics without IDA.

This is This is consistent with the results of Kim et al. [23] who observed IDA more frequently in infants whose nutrition was supplied only by breastfeeding for more than 6 months, in those who were fed weaning food with low iron content and in those who took a long time to adapt to the weaning food [23].

This is similar to Al Ghwass et al. [24] who studied 345 children aged 6 months to 12 years and found that the frequency of IDA was $64 \%$ among them. This high prevalence of iron deficiency among study population explained by the consumption of unfortified cow's milk feeding during the 1 year of life, low intake of iron-rich foods, unmet increased needs for iron due to rapid growth.

While the prevalence of IDA was higher in infants fed with the formula without iron (20\%), much lower in those fed with iron-fortified formula $(0.6 \%)$, and medium in infants fed with human milk $(15 \%)$. In another study, an increased prevalence of IDA in infancy was observed in infants fed with nonformula cow's milk $>600 \mathrm{ml}$ or more daily or $>6$ breast feeds per day [25]

There was a significant positive correlation between increased number of meals containing animal meat, chicken meat, liver, and IDA. This is in agreement with Mamdooh; there was a significant association between the consumption of food rich in iron and IDA. This can be as attributed to the fact that liver and meat are very rich in heme iron [18]

\section{Diabetes complications:}

\section{Acute complication:}

The current study found that the presence of hypoglycemic coma, DKA were significantly higher 
in diabetics with iron deficiency anemia than in diabetics without IDA this is consistent with Soliman et al. [2] who found that Iron deficiency (ID) and IDA can impair glucose homeostasis in human and negatively affect glycemic control and predispose to more complications like hypoglycemia and diabetic ketoacidosis in diabetic patients.

\section{Chronic complication:}

The present study revealed that the frequency of neuropathy in children with T1DM was $26.5 \%$ $(53 / 200)$.

Similarly the European Diabetes Prospective Complications Study reported that the neuropathy prevalence was $28 \%$ at baseline [26]

On the other hand a study at Zagazig University Hospitals revealed that the frequency of neuropathy in children with T1DM was $42.5 \%$ (17/40) [27]. In addition, a study conducted by Moser and coworkers reported that of 151 youth with type I diabetes $11 \%$ were diagnosed with diabetic peripheral neuropathy (DPN).

This difference in frequency of neuropathy in diabetic patient may be due to difference in duration of diabetes among study populations.

In our study we found that the frequency of diabetic nephropathy in children with T1DM was $15.5 \%(31 / 200)$.

While $25 \%$ to $40 \%$ of patients with T1D develop diabetic kidney disease approximately $20 \%$ to $30 \%$ of T1D have microalbumiuria after mean diabetes duration of 15 years and the overall incidence of end stage renal disease is reported to be $4 \%$ to $17 \%$ at 20 to 30 years from T1D diagnosis [28].

While on the other hand in 86 patients, prevalence of micro albuminuria was $6 \%$ [29].

While Diabetic nephropathy was diagnosed in 10 patients (31\%), 2 with microalbuminuria and 8 with proteinuria [30]

This variation in prevalence rates for micro albuminuria might be explained by differences in study populations, such as age range, diabetes duration, glycemic control, and length of followup ranging.

In our study, we have demonstrated the prevalence of retinopathy to be $6.5 \%$ (13/200) for any retinopathy in children and adolescents while Klein et al. [31] demonstrated that the prevalence of retinopathy was less than $10 \%$ during the first 5 years after onset of type I diabetes, in children younger than 13 years. While Kernell et al. [32] a have demonstrated the prevalence of retinopathy to be $14.5 \%$ for any retinopathy and $2.3 \%$ for proliferative and preproliferative retinopathy in children and adolescents.

\section{Parasitic infection:}

The prevalence of parasitic infestation among our 200 patient was $24 \%$ (48/200) while the prevalence of intestinal parasites in 1920 school-age children from eight schools located in Thailand was presented in Table (1). The overall prevalence of intestinal parasites was $12.6 \%(242 / 1920)$ [33]

A previous study conducted in Jeddah, KSA reported that the overall prevalence of the parasitic infection was 48\%, A study conducted in Morocco showed that the mono- or polyparasitism was detected in $34.5 \%$ of the children [34]

The high prevalence of intestinal parasites recorded in the study could be attributed to exposure of the children to predisposing factors to intestinal parasitic infections; such as (poor sewage disposal system, unsafe sources of water, poor sanitary conditions, poor housing and lack of awareness on the part of the parents and children).

The prevalence of IDA among children with a positive medical history for diarrhea and parasitic infestation was higher than that among children with a negative medical history for diarrhea and parasitic infestation. This result is in agreement with Abdel-Rasoul et al., diarrheal and parasitic infestations were reported in different studies in the Gaza Strip and have been shown to be associated with anemia among school-age children in Gaza. Also Abdel-Rasoul et al. [18] who found that of IDA among children with a positive medical history for diarrhea and parasitic infestation was higher than that among children with a negative medical history for diarrhea and parasitic infestation (59.9 and $80.3 \%$, respectively). This may be because diarrhea and parasitic infestation affect absorption and may lead to loss of blood from gastro intestinal tract (GIT).

\section{Anthropometric measures:}

In this study, mean BMI in IDA diabetic group was low compared to non-IDA diabetic group 17.99 $\pm 3.77,20.60 \pm 5.20 \mathrm{~kg} / \mathrm{m}^{2}$ patients with highly significant statistical difference ( $p$-value $=0.001)$. Also patients with IDA were shorter and lighter than non-anemic patient but this didn't reach statistical significance. 
Similarly Al Ghwass et al. [24] showed that stunting, wasting and underweight were associated among iron deficient anemic children, but underweight only was statistically significant.

This agrees with Luo et al., who reported that IDA during the first 2 years of life significantly impairs growth, and there is a significantly correlation between growth velocity and SF concentration. Also Luo et al. [35] mentioned that children with anemia were shorter for their age, and a higher percentage of them of them had stunted growth.

\section{Clinical data:}

In our study the mean heart rate was significantly higher among cases with IDA than those without IDA (91.82 \pm 7.26 bpm vs. / 88.50 \pm 8.47 bpm). Similarly Mustafa et al. [36] found the same results the mean heart rate was significantly higher among anemic patients compared to the control group ( $84.4 \pm 22.4 \mathrm{bpm}$ vs. $72.6 \pm 13.2 \mathrm{bpm} ; p=0.005$.

In this study presence of pallor was highly significantly higher in diabetic children with iron deficiency than those without iron deficiency anemia.

This is consistent with the results of Stoltzfus et al. [37] that studied five different study samples and provide information on the performance of clinical pallor to detect low hemoglobin based on observations of 5,760 individuals, 3,072 of whom were anemic, and 192 of whom had hemoglobin concentrations $<70 \mathrm{~g} / \mathrm{Land}$ found that Hemoglobin concentration significantly lower in group with pallor compared to without pallor for all sites, $p<0.001$.

Similarly, This has been a consistent finding in all of the published studies of the clinical assessment of anemia that we were able to identify [38,39]

\section{Laboratory result:}

The present study revealed that mean $\mathrm{HbA} 1 \mathrm{c}$ is higher in IDA group (10.16 \pm 1.23$)$ compared to the group without IDA $(8.84 \pm 0.94)(p<0.000)$.

Similarly a prospective study including 37 patients with type I diabetes (1 1 patients were ID and the remaining 26 were iron sufficient). Patients with ID had higher levels of HbA1c than patients without iron deficiency [2].

Also in 200 type I diabetic patients attending a pediatric diabetic clinic in Cairo, HbA1c levels were statistically significantly higher in iron- deficient than in non-iron-deficient patients $(p<0.01)[15]$

This is supported by studies done by, Ford et al., in [11], Silva et al., in [41], have obtained higher HbA1c level in IDA patients.

In contrary Solomon et al. [20] revealed that HbA1c (\%) is significantly lower in IDA group $(6.18 \pm 1.57)$ compared to the control group (7.74 $\pm 1.81)(p<0.05)$. This is supported by studies done by Sinha et al., in [42], Cavagnolli et al., in [43]

They all stated that HbAlc concentration tends to be lower in the presence of iron deficiency anemia. According to Sinha et al. [42] suggestion, the reason for lower $\mathrm{HbA} 1 \mathrm{c}$ is due to the severity of anemia in the study participants.

Christy et al., in [44] found a positive correlation between IDA and increased A1C levels.

The present study revealed that mean cell volume is lower in IDA group (65.96 \pm 6.44$)$ compared to non-IDA group $(79.38 \pm 5.74)$ this result was highly significant ( $p$-value 0.000 ).

Similarly Solomon et al. [20] mean hemoglobin, mean cell volume (MCV), was lower in IDA group compared to non-IDA diabetic patients.

In this study, median hepcidin was lower in IDA group compared to non-IDA diabetic children 1000 (750-2100), 2900 (2450-3180) ug/ml with highly significant statistical difference.

Similarly a study evaluated serum hepcidin concentrations in relation to iron status in 215 children with T1D found that: In patients with absolute iron deficiency there is lower hepcidin concentrations compared to patients with normal iron status. $(p$-value $=0.000)[13]$.

This is consistent with the results of Choi et al. [45] who studied 59 children (23 males and 36 females) and found that mean serum hepcidin levels were significantly lower in the ID $(7.72 \pm 8.03$ $\mathrm{ng} / \mathrm{mL})$ and IDA groups $(2.01 \pm 2.30 \mathrm{ng} / \mathrm{mL})$. Low serum hepcidin in ID and IDA can be attributed to the lower total iron stores in these groups than that in the normal controls.

Similarly Girelli et al. [46] found the same results In IDA, hepcidin levels are generally suppressed.

In addition D'Angelo [47] found the same results In pure iron deficiency anemia (IDA), serum and urinary hepcidin concentrations are significantly 
decreased and even in the absence of anemia, hepcidin appears to be a sensitive indicator of iron deficiency. Moreover, compared to hematocrit or hemoglobin, a decrease in hepcidin is an early marker of iron deficiency together with transferrin saturation and decreased ferritin.

In our study, mean serum ferritin was lower in IDA group compared to non-IDA diabetic 45 (20$80), 50(32.5-60) \mathrm{ng} / \mathrm{ml}$ patients but with no significant statistical difference $(p$-value $=0.350)$.

This is consistent with the results of Madanat [48] mean serum ferritin for the iron deficiency anemia group was $39.1 \mathrm{ng} / \mathrm{mg}$ as compared to $84.7 \mathrm{ng} / \mathrm{ml}$ for the normal group. Even though the serum ferritin level was lower in the iron deficiency group, the difference in the means did not reach statistical significance.

Similarly Bouri and Martin [49] found the same results In IDA the ferritin, serum iron and transferrin saturations are low, but the TIBC increases.

\section{Summary:}

Iron deficiency anemia a prevalent finding in patients with type I diabetes and represents a significant unrecognized burden.

In this study, we aim to study prevalence of iron deficiency anemia among type I diabetic children and to identify possible etiologies of iron deficiency anemia and to correlate parameters to glycemic control.

This is particularly important as iron deficiency anemia is associated with impaired psychomotor and mental development in infants, and neurocognitive impairment in adolescents.

This case-control study included two hundred children with type I diabetes recruited from Pediatrics and Adolescent Diabetes Unit (PADU), Ain shams university in the period from December 2019 to July 2020.

Cases (123 males and 77 females) are children with type I diabetes. Diagnosis of type I diabetes are based upon criteria of ISPAD 2018. Their mean age was $10.97 \pm 3.93$ years (Range: $2-18$ years).

Cases were subjected to full history taking; general examination with laying stress on heart rate, pallor and anthropometric measurements and laboratory evaluation including complete blood count, glycated haemoglobin (HbA1c) and Patients with microcytic hypochromic anaemia underwent assessment of:
Serum iron, total iron-binding capacity (TIBC) and serum ferritin, Hepcidin, Anti-tissue transglutaminase $(\operatorname{Ig} \mathrm{A})$, Occult blood in stool and $\mathrm{H}$ pylori antigen in stool.

Our results showed upon comparison of cases with IDA to those without IDA: Cases with IDA had significantly higher values as regards male sex, lower age, hypoglycemic attacks, DKA attacks, menorrhagia history, higher fatigue severity scale, neuropathy, tachycardia, pallor, lower MCV, lower body weight, lower BMI, higher TIBC and lower hepcidin level.

Also longer diabetes duration, higher insulin dosage retinopathy, higher RDW, lower Hba1c, lower height and h-pylori antigen in stool are higher in cases with IDA than in cases without IDA with no statistical difference.

$36 \%$ of diabetic patient were anemic and $25.5 \%$ had iron deficiency anemia.

Our study has several limitations. The study sample was small and based solely on diabetic children who were recruited from our clinic. This study used a case control study design so that the causality between iron deficiency anemia and type I diabetes could not be established, just the association between them that could be suggested.

\section{Conclusions:}

The incidence of anemia in the early stages of the disease justifies conducting the screening in all children with type I diabetes and taking appropriate preventive measures toward the patients at risk for iron-deficiency anemia.

Serum hepcidin levels are significantly associated with iron status in children, and could be useful indicators of ID. Further studies are necessary to confirm the value of serum hepcidin measurement in the diagnosis of ID and to determine the reliable reference range and cutoff values in children.

\section{References}

1- RUSAK E., ROTARSKA-MIZERA A., ADAMCZYK P., MAZUR B., POLANSKA J. and CHOBOT A.: Markers of Anemia in Children with Type 1 Diabetes. Journal of Diabetes Research, 5184354, 2018.

2- SOLIMAN A.T., De SANCTIS V., YASSIN M. and SOLIMAN N.: Iron deficiency anemia and glucose metabolism. Acta Bio Medica: Atenei Parmensis, 88 (1): 112, 2017.

3- THOMAS M.C., MACISAAC R.J., TSALAMANDRIS C., MOLYNEAUX L., GOUBINA I., FULCHER G., YUE D. and JERUMS G.: Anemia in patients with type 1 diabetes. The Journal of clinical endocrinology and metabolism, 89 (9): 4359-4363, 2004. 
4- BUTTARELLO M. and PLEBANI M.: Automated blood cell counts: State of the art, American Journal of Clinical Pathology, 130 (1): 104-16, 2008.

5- Journal of the Egyptian Public Health Association, 2015.

6- KRUPP L.B., LaROCCA N.G., MUIR-NASH J., STEINBERG A.D.: The fatigue severity scale. Application to patients with multiple sclerosis and systemic lupus erythematosus. Arch. Neurol., 46 (10): 1121-3, 1989.

7- EL SHAFIE A.M., EL-GENDY F.M., ALLAHONY D.M., OMAR Z.A., SAMIR M.A., EL-BAZZAR A.N., ABD EL-FATTAH M.A., ABDEL MONSEF A.A., KAIRALLAH A.M., RAAFET H.M., BAZA G.M., SALAH A.G., GALAB W.S., KASEMY Z.A. and BAHBAH W.A.: Establishment of Z Score Reference of Growth Parameters for Egyptian School Children and Adolescents Aged From 5 to 19 Years: A Cross Sectional Study. Frontiers in Pediatrics, 8: 368, 2020.

8- NAH E., KIM S., CHO S. and CHO H.: Complete Blood Count Reference Intervals and Patterns of Changes Across Pediatric, Adult, and Geriatric Ages in Korea. Annals of Laboratory Medicine, 38 (6): 503-511, 2018.

9- GOMELLA L.G. and HAIST S.A.: Clinician's Pocket Reference: The Scut Monkey. 11 ed. New York, 2007.

10- DIETERICH W., EHNIS T., BAUER M., DONNER P., VOLTA U., RIECKEN E.O. and SCHUPPAN D.: Identification of tissue transglutaminase as the autoantigen of celiac disease. Nat. Med., 3 (7): 797-801, 1997.

11-HAREWOOD G.C., McCONNELL J.P., HARRINGTON J.J., MAHONEY D.W. and AHLQUIST D.A.: Detection of occult upper gastrointestinal tract bleeding: Performance differences in fecal occult blood tests. Mayo. Clin. Proc., 77 (1): 23-8, 2002.

12- SELVARAJAH D., CASH T., DAVIES J., SANKAR A., RAO G., GRIEG M., PALLAI S., GANDHI R., WILKINSON I.D. and TESFAYE S.: SUDOSCAN: A Simple, Rapid, and Objective Method with Potential for Screening for Diabetic Peripheral Neuropathy. PloS One, 10 (10): e0138224, 2015.

13- MIRJAM V., MARJOLIJN D.A., RACHEL P.L., COBY M.L., EUPHEMIA C.A.M., BOUDEWIJN B., AGNES C., DANIËLLE C.M., MARTINE C.V., CLAIRE W., DICK M., JOHANNES B.G. and FRANK B.: Serum hepcidin concentrations in relation to iron status in children with type 1 diabetes, Pediatric Hematology and Oncology, 38 (2): 108-123, 2021.

14- WÓJCIAK R., MOJS E. and STANISLAWSKA-KUBIAK M.: The Occurrence of Iron-Deficiency Anemia in Children With Type 1 Diabetes. Journal of investigative medicine: The official publication of the American Federation for Clinical Research, 62, 2014.

15- SALAH N., ABDEL HAMID F., ABDEL GHAFFAR S. and EL SAYEM M.: Prevalence and type of anaemia in young Egyptian patients with type 1 diabetes mellitus. EMHJ-Eastern Mediterranean Health Journal, 11 (5-6): 959-967, 2005.

16- BERGIS D., TESSMER L. and BADENHOOP K.: Iron deficiency in long standing type 1 diabetes mellitus and its association with depression and impaired quality of life. Diabetes research and clinical practice, 151: 74-81, 2019.
17- AKKERMANS M.D., MIEKE HOUDIJK E.C.A. and BAKKER B.: Iron status and its association with HbA1c levels in Dutch children with diabetes mellitus type 1 Eur. J. Pediatr., 177: 603-610, 2018.

18- ABDEL-RASOUL G.M., EL BAHNASY R.E., EL SHAZLY H.M., GABR H.M. and ABDEL-AATY N.B.: Epidemiology of iron-deficiency anemia among primary school children (6-11 years), Menoufia governorate, Egypt. Menoufia Medical Journal, 28 (3): 663, 2015.

19- NEUMAN N., TANAK O., SZARFAR S. and VICTORA G.: Prevalence and risk factors for anemia in Southern Brazil. Rev. Saudi Publica, 34: 56-63, 2000.

20- SOLOMON A., HUSSEIN M., NEGASH M., et al.: Effect of iron deficiency anemia on $\mathrm{HbA} 1 \mathrm{c}$ in diabetic patients at Tikur Anbessa specialized teaching hospital, Addis Ababa Ethiopia. BMC Hematol 19: 2, 2019.

21- ANDRIASTUTI M., ILMANA G., AVILIA S. and KOSASIH K.A.: Prevalence of anemia and iron profile among children and adolescent with low socio-economic status, International Journal of Pediatrics and Adolescent Medicine, 7 (2): 88-92, 2019.

22- MOHAMED E., AHMED O. and YOUSSEF A.: Iron deficiency and anemia in rural school children in a Coastal Area of Morocco. Pak. J. Nutr., 7: 400-403, 2008.

23- KIM H.J., KIM D.H., LEE J.E., KWON Y.S., JUN Y.H., HONG Y.J. and KIM S.K.: Is It Possible to Predict the Iron Status from an Infant's Diet History?. Pediatric gastroenterology, hepatology \& nutrition, 16 (2): 95-103, 2013.

24- AL GHWASS M.M., HALAWA E.F., SABRY S.M. and AHMED D.: Iron deficiency anemia in an Egyptian pediatric population: a cross-sectional study. Annals of African Medicine, 14 (1): 25-31, 2015.

25- HARE D.J., CARDOSO B.R., SZYMLEK-GAY E.A. and BIGGS B.A.: Neurological effects of iron supplementation in infancy: finding the balance between health and harm in iron-replete infants. The Lancet Child and Adolescent Health, 2 (2): 144-156, 2018.

26- TESFAYE S., CHATURVEDI N., EATON S.E.M., WARD J.D., MANES C. and IONESCU-TIRGOVISTE C.: Vascular Risk Factors and Diabetic Neuropathy. N. Engl. J. Med., 352: 341-50, 2005.

27- AMER M. and KAMAL H.: Study of peripheral neuropathy in children with type 1 diabetes mellitus at zagazig university hospitals. Zagazig University Medical Journal, 25: 116-125, 2019.

28- PAPADOPOULOU-MARKETOU N., CHROUSOS G.P. and KANAKA-GANTENBEIN C.: Diabetic nephropathy in type 1 diabetes: A review of early natural history, pathogenesis, and diagnosis. Diabetes Metab. Res. Rev., 33: e2841, 2017

29- BOGDANOVI'C R.: Diabetic nephropathy in children and adolescents. Pediatr. Nephrol., 23: 507-525, 2008.

30- AL-HERMI B.E., AL-ABBASI A.M., RAJAB M.H., ALJENAIDI F.A. and AL-EKRI Z.E.: Diabetic nephropathy in children with type 1 diabetes mellitus in Bahrain. Saudi Medical Journal, 26 (2): 294-297, 2005.

31- KLEIN R., KLEIN B.E., MOSS S.E., DAVIS M.D. and De METS D.L.: Retinopathy in young-onset diabetic patients. Diabetes Care, 8: 311-315, 1985. 
32- KERNELL A., DEDORSSON I., JOHANSSON B., et al.: Prevalence of diabetic retinopathy in children and adolescents with IDDM A population-based multicenter study. Diabetologia, 40: 307-310, 1997.

33- NGRENNGARMLERT W., LAMOM C., PASURALERTSAKUL S., YAICHAROEN R., WONGJINDANON N., SRIPOCHANG S., SUWAJEEJARUN T., SERMSART B.O. and KIATFUENGFOO R.: Intestinal parasitic infections among school children in Thailand. Trop Biomed, 24 (2): 83, 2007.

34- MESSAAD S.A., LABOUDI M., MOUMNI M., SARHANE B., BELGHYTI D. and EL KHARRIM K.H.: Children intestinal parasites related to socioeconomic factors in Salé hospital, Morocco. Int. J. Innov. Appl Stud., 8 (2): 833-840, 2014.

35- LUO R., ZHANG L., LIU C., ZHAO Q., SHI Y. and MILLER G.: Anaemia among students of rural China's elementary schools: Prevalence and correlates in Ningxia and Qinghai's poor counties. J. Health Popul. Nutr., 29: 471-85, 2011.

36- MUSTAFA T., YILMAZ G., UNAL G., ALI G.H., BEYHAN E., NIYAZI G., et al.: Heart rate variability in patients with iron deficiency anemia. Arq. Bras. Cardiol., [cited 2021 Mar 20]; 92 (5): 400-403, 2009.

37- STOLTZFUS R.J., EDWARD-RAJ A., DREYFUSS M.L., ALBONICO M., MONTRESOR A., DHOJ THAPA M., WEST Jr K.P., CHWAYA H.M., SAVIOLI L. and TIELS$\mathrm{CH}$ J.: Clinical pallor is useful to detect severe anemia in populations where anemia is prevalent and severe. The Journal of Nutrition, 129 (9): 1675-81, 1999.

38- GJORUP T., BUGGE P.M., HENDRIKSEN C. and JENSEN A.M.: A critical evaluation of the clinical diagnosis of anemia. Am. J. Epidemiol., 124: 657-665, 1986.

39- KALTER H.D., BURNHAM G., KOLSTAD P.R., HOSSAIN M., SCHILLINGER J.A., KHAN N.Z., SAHA S., De WIT V., KENYA-MUGISHA N., SCHWARTZ B. and BLACK R.E.: Evaluation of clinical signs to diagnose anaemia in Uganda and Bangladesh, in areas with and without malaria. Bulletin of the World Health Organization, 75 (Suppl 1): 103, 1997.

40- FORD E.S., COWIE C.C., LI C., HANDELSMAN Y. and BLOOMGARDEN Z.T.: Iron-deficiency anemia, noniron-deficiency anemia and $\mathrm{HbA} 1 \mathrm{c}$ among adults in the US. J. Diabete, 3 (1): 67-73, 2011.

41- SILVA J.F., PIMENTEL A.L., SILVA J.F. and CAMARGO J.L.: Effect of iron deficiency anemia on HbA1c levels is dependent on the degree of anemia. Clin. Biochem. 10.1016/j.clinbiochem.2015.09.004, 2015.

42- SINHA N., MISHRA T.K., SINGH T. and GUPTA N.: Effect of Iron deficiency Anemia on hemoglobin A1c levels. Ann. Lab. Med., 32 (1): 17-22, 2012.

43- CAVAGNOLLI G., PIMENTEL A.L., FREITAS P.A.C., GROSS J.L. and CAMARGO J.L.: Factors affecting A1C in non-diabetic individuals: review and meta-analysis. Clin. Chim. Acta., 445: 107-14, 2015.

44- CHRISTY A.L., MANJREKAR P.A., BABU R.P., HEGDE A. and RUKMINI M.S.: Influence of Iron Deficiency Anemia on Hemoglobin A1 C Levels in Diabetic Individuals with Controlled Plasma Glucose Levels. Iran Biomed J., 18: 88-93, 2014.

45- CHOI H.S., SONG S.H., LEE J.H., KIM H.J. and YANG H.R.: Serum hepcidin levels and iron parameters in children with iron deficiency. The Korean Journal of Hematology, 47 (4): 286-292, 2012.

46- GIRELLI D., NEMETH E. and SWINKELS D.W.: Hepcidin in the diagnosis of iron disorders. Blood, 127 (23): 2809-2813, 2016.

47- D'ANGELO G.: Role of hepcidin in the pathophysiology and diagnosis of anemia. Blood Research, 48 (1): 10-15, 2013.

48- MADANAT F., EL-KHATEEB M., TARAWANEH M. and HIJAZI S.: Serum ferritin in evaluation of iron status in children. Acta. Haematologica, 71 (2): 111-115, 1984.

49- BOURI S. and MARTIN J.: Investigation of iron deficiency anaemia. Clinical Medicine, 18 (3): 242, 2018. 


\section{أنيميا نقص الحديد فى الأطفال والمراهقين المصابين بمرض السكر من النوع الأول}

يعد فقر الدم الناجم عن نقص الصديد العبب الرئيسى لفقر الدم فى الأطفال كما يعتبر تصدياً عالمياً. فما يقرب من • ع٪ من الأطفال مصابون

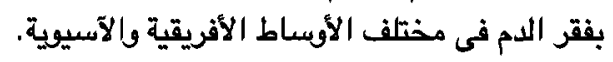
في هذه الدراسة، نهدف إلى دراسة معدل انتشار ذقر الدم الناجم عن نقص الحديد بين الأطفال المصابين بالسكرى من النوع الأهل وتحديد

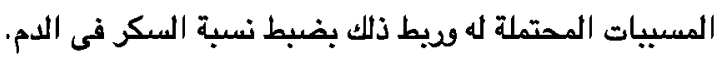

وهذا في غاية الأهمية حيث أن فقر الدم الناجم عن نقص الصديد يرتبط بخل فى النمو الصركى والعقلى عند الرضع، وضعف الإدرال العصبى لدى المراهقين.

ولقد تضمنت هذه الدراسة مائتى طفل يعانوف من مرض السكرى من النوع الأفل تم تجميعهم من وحدة طب الأطفال ومرض السكرى لدى الى

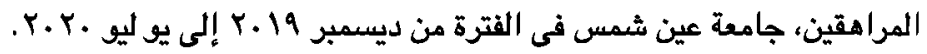
هذه الدراسة تشمل مائتى طفل (بrا من الذكو و VV من الإناث) هم أطفال يعانون من داء السكرى من النوع الأفل، ويستند تشخيص

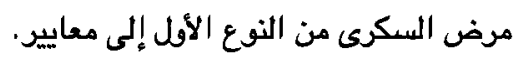

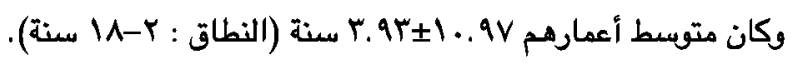

وقد خضعت الحالات لأخذ تاريخ مرضى كامل، وفحص عام الجسم مع التركيز على معدل ضربات القلب، والثحوب والقياسات البشرية

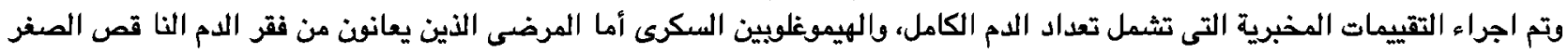
خضعوا لتقييمات اضعافية وتشمل :

نسبة الحديل بالدم، القدرة الكلية على الارتباط بالحديل ونسبة الفيريتين بالدم، فحص حساسية الجلوتين، الهبسيدين، الدم الخفى فى البراز ومستضد جرثومه المعدة في البراز.

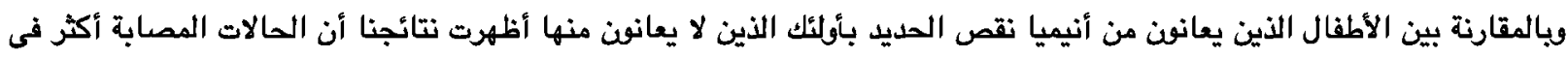

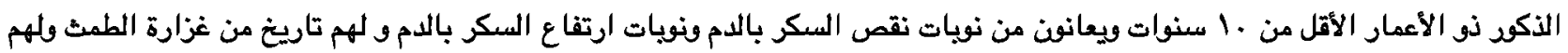

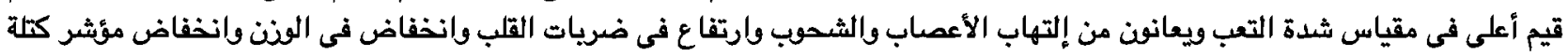

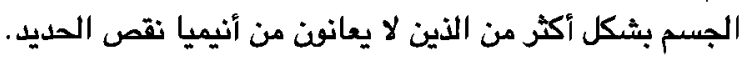

كما وجد أن مستوى الهبسيدين في الدم أقل ومتوسط حجم كريات الدم الحمراء أقل والقدرة الكلية على الارتباط بالحديد أعلى فى أولئك الذين يعانون من أنيميا نقص الحديد.

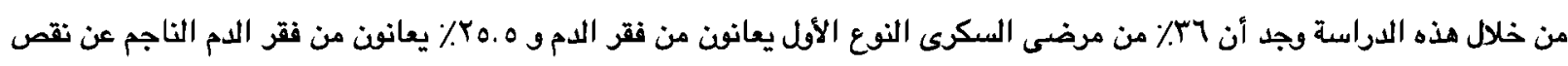

